\title{
Cancer gene therapy using a survivin mutant adenovirus
}

\author{
Mehdi Mesri, ${ }^{1}$ Nathan R. Wall, ${ }^{1}$ Jia Li, ${ }^{1}$ Richard W. Kim, ${ }^{2}$ and Dario C. Altieri ${ }^{1}$ \\ ${ }^{1}$ Department of Pathology, and \\ ${ }^{2}$ Department of Surgery, Boyer Center for Molecular Medicine, Yale University School of Medicine, New Haven, Connecticut, USA \\ Address correspondence to: Dario C. Altieri, Yale University School of Medicine, BCMM436B, 295 Congress Avenue, \\ New Haven, Connecticut 06536, USA. Phone: (203) 737-2869; Fax: (203) 737-2402; E-mail: dario.altieri@yale.edu.
}

Received for publication April 10, 2001, and accepted in revised form August 7, 2001.

\begin{abstract}
We have constructed a replication-deficient adenovirus encoding a nonphosphorylatable $\mathrm{Thr}^{34} \rightarrow \mathrm{Ala}$ mutant of the apoptosis inhibitor survivin (pAd-T34A) to target tumor cell viability in vitro and in vivo. Infection with pAd-T34A caused spontaneous apoptosis in cell lines of breast, cervical, prostate, lung, and colorectal cancer. In contrast, pAd-T34A did not affect cell viability of proliferating normal human cells, including fibroblasts, endothelium, or smooth muscle cells. Infection of tumor cells with pAd-T34A resulted in cytochrome $c$ release from mitochondria, cleavage of approximately 46-kDa upstream caspase- 9 , processing of caspase- 3 to the active subunits of approximately 17 and $19 \mathrm{kDa}$, and increased caspase-3 catalytic activity. When compared with chemotherapeutic regimens, pAd-T34A was as effective as taxol and considerably more effective than adriamycin in induction of tumor cell apoptosis and enhanced taxol-induced cell death. In three xenograft breast cancer models in immunodeficient mice, pAd-T34A suppressed de novo tumor formation, inhibited by approximately $40 \%$ the growth of established tumors, and reduced intraperitoneal tumor dissemination. Tumors injected with pAd-T34A exhibited loss of proliferating cells and massive apoptosis by in situ internucleosomal DNA fragmentation. These data suggest that adenoviral targeting of the survivin pathway may provide a novel approach for selective cancer gene therapy.
\end{abstract}

J. Clin. Invest. 108:981-990 (2001). DOI:10.1172/JCI200112983.

\section{Introduction}

The coupling of apoptosis to cellular surveillance mechanisms, that is, checkpoints, is thought to provide a critical safeguard against the development of cancer (1). Defects in apoptotic pathways (2) with failure to eliminate cells harboring DNA damage, viral infection, or abnormalities in the mitotic apparatus have been linked to the onset and progression of cancer (3), and mutations in proapoptotic genes may confer increased tumor invasiveness and resistance to therapy (4). Among the regulators of apoptotic pathways, bcl-2 proteins have been implicated in the control of mitochondrial homeostasis, most notably cytochrome $c$ release (5), whereas members of the inhibitor of apoptosis (IAP) gene family control a downstream step in cell death by suppressing processing and catalytic activity of caspases, the executioners of cell death programs (6).

A role for IAP proteins in cancer has been recently highlighted with the identification of the survivin gene (7). Survivin is a structurally unique IAP family protein that is expressed in mitosis in a cell cycle-dependent fashion and localized to components of the mitotic apparatus (8). Potentially involved in both inhibition of apoptosis and control of cell division (9), survivin expression is prominently upregulated in most human cancers, but undetectable or found at very low levels in normal adult tissues $(7,10)$. In several tumor series, the presence of survivin correlated with reduced apop- totic index in vivo, abbreviated overall survival, unfavorable prognosis, and accelerated rates of recurrences (11-15). In addition, the integrity of the survivin pathway may be required for cancer cell viability. Molecular antagonists of survivin including antisense or expression of a dominant negative mutant resulted in spontaneous apoptosis of cancer cells in vitro $(16,17)$, and in vivo (18), and enhancement of chemotherapyinduced cell death (19).

Because manipulation of apoptosis may provide new therapeutic strategies in human diseases (20), we sought to target the survivin pathway in cancer with a replication-deficient adenovirus encoding a survivin $\mathrm{Thr}^{34} \rightarrow$ Ala mutant, which abolishes a phosphorylation site for $\mathrm{p} 34^{\mathrm{cdc} 2}-\mathrm{cyclin} \mathrm{B} 1$ (21). Here, we show that the survivin mutant virus caused initiation of the mitochondrial apoptotic pathway in various tumor cell types, exhibited no toxicity for normal human cells, and suppressed tumor growth in three different xenograft breast cancer models in vivo.

\section{Methods}

Cells and cell cultures. Human cervical carcinoma HeLa, breast carcinoma MCF-7, lung carcinoma A549, prostate carcinoma PC3, colorectal carcinoma HCT116, and embryonic kidney 293 cells were from American Type Culture Collection (Manassas, Virginia, USA) and maintained in culture according to the supplier's 
a

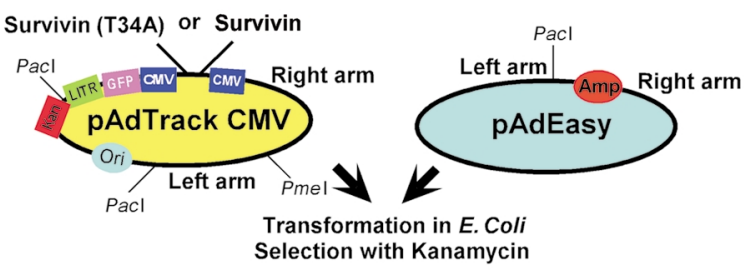

b

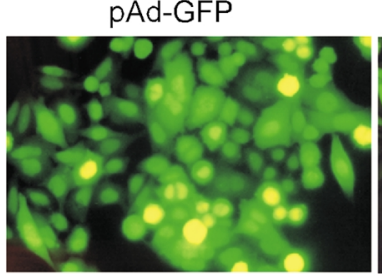

c
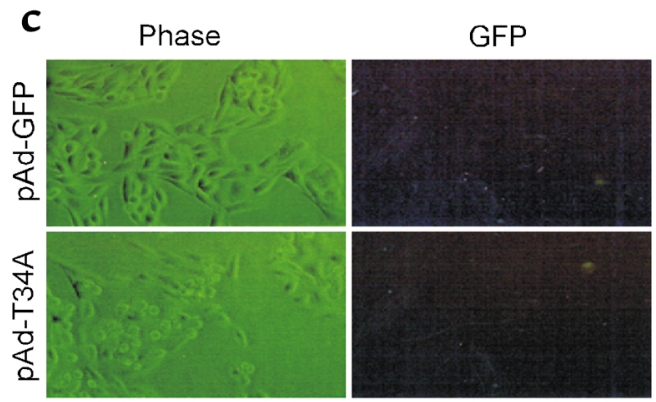

pAd-WT

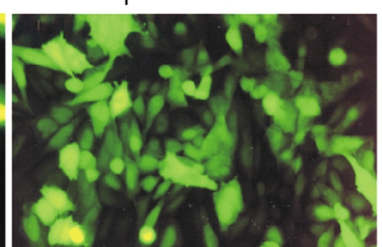

d

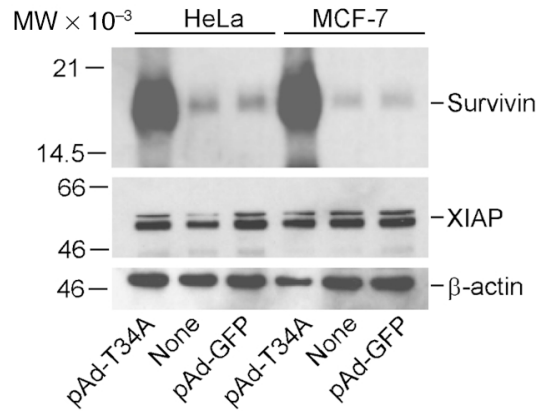

\section{Figure 1}

Construction and expression of pAd survivin vectors. (a) Map of pAdTrack CMV and pAdEasy vectors. (b) GFP expression in transduced cultures. HeLa cells were infected with the indicated pAd vectors at moi of 50 for 8 hours, harvested after 48 hours, and analyzed by fluorescence microscopy. (c) Absence of replication-competent adenoviral particles. HeLa cells $\left(8 \times 10^{4}\right)$ were infected with pAd-T34A or pAdGFP at moi of 1,250 and grown for 3 days at $37^{\circ} \mathrm{C}$. Cell extracts were used to successively infect a second HeLa cell culture, and cells were analyzed by phase-contrast microscopy (Phase) or GFP expression (GFP) after an additional 2-day period. (d) Western blot analysis. Aliquots of HeLa or MCF-7 cells were infected with the indicated pAd vectors at moi of 50 , harvested after 48 hours at $37^{\circ} \mathrm{C}$, and proteinnormalized extracts were analyzed by Western blotting with an $A b$ to survivin, $\mathrm{XIAP}$, or control $\beta$-actin followed by chemiluminescence. Molecular-weight markers in kilodaltons are shown on the left. LITR, left-hand inverted terminal repeat; MW, molecular weight. specifications. Normal human lung fibroblasts were obtained from Clonetics Corp. (San Diego, California, USA) and grown in FGM2 medium. Normal human vascular smooth muscle cells (VSMCs) were obtained from Coriell Cell Repositories (Camden, New Jersey, USA) and maintained in 199 medium containing 10\% FCS, $0.02 \mu \mathrm{g} / \mathrm{ml}$ endothelial cell growth supplement, and $0.05 \mu \mathrm{g} / \mathrm{ml}$ heparin. Normal human foreskin fibroblasts (HFFs) were obtained from ATCC and grown in complete DMEM containing $10 \%$ FCS. Human umbilical vein endothelial cells (HUVECs) were isolated and maintained in culture as described (22).

Construction of adenoviral vectors. The adenoviral (pAd) vectors for homologous recombination in bacteria have been described (23) (Figure 1a). Both pAdTrack-CMV and pAdEasy-1 vectors were kindly provided by B. Vogelstein (Johns Hopkins University School of Medicine, Baltimore, Maryland, USA). The cDNAs for wildtype (WT) survivin or $\mathrm{Thr}^{34} \rightarrow$ Ala survivin mutant (T34A) containing $5^{\prime}$ HindIII and $3^{\prime} X b a I$ sites were inserted in pAdTrack downstream of the cytomegalovirus (CMV) promoter (Figure 1a) to generate pAd-WT or pAd-T34A. Each shuttle vector was linearized with PmeI, electroporated in Escherichia coli BJ5183, and colonies were selected in $50 \mu \mathrm{g} / \mathrm{ml}$ of kanamycin. Each pAd construct $(4-10 \mu \mathrm{g})$ was digested with PacI, transfected in 293 cells by Lipofectamine (Life Technologies Inc., Rockville, Maryland, USA), and cultures were monitored for expression of green fluorescent protein (GFP), by fluorescence microscopy. The cell pellets were suspended in $1 \mathrm{ml}$ PBS, $\mathrm{pH} 7.4$, and after three cycles of freezing and thawing, $1 \mathrm{ml}$ of viral lysate supernatant was used to infect $3 \times 10^{6}$ to $5 \times 10^{6}$ 293 cells. Viruses were harvested at 2- to 3-day intervals. To generate high-titer viral stocks, this process was repeated 3-5 times with a total of $5 \times 10^{8}$ packaging cells, and viral particles were purified by $\mathrm{CsCl}$ banding. Green fluorescence forming units (GFU) were estimated by serial dilution of the virus stock in transduced 293 cells. To detect a potential contamination of viral stocks with replication-competent adenoviral particles, HeLa cells $\left(8 \times 10^{4}\right)$ in C-6-well plates were infected with pAd-GFP or pAd-T34A at moi of 1,250 for 8 hours and grown for 3 days at $37^{\circ} \mathrm{C}$. Cell extracts prepared by freezing and thawing and supernatants were used to successively infect a second culture of HeLa cells, which was analyzed for viral transduction by GFP expression after an additional 2-day interval at $37^{\circ} \mathrm{C}$. Expression of survivin protein in transduced cultures was determined by Western blot analysis, using an $\mathrm{Ab}$ to fulllength recombinant survivin (NOVUS Biologicals Inc., Littleton, Colorado, USA), as described (24).

Apoptosis and cell cycle analysis. Subconfluent cultures of the various tumor cell types or normal human primary cells were incubated with pAd-GFP, pAd-WT, or pAd$\mathrm{T} 34 \mathrm{~A}$ at moi of 50 for 8 hours at $37^{\circ} \mathrm{C}$. After additional 48-hour incubation in complete medium at $37^{\circ} \mathrm{C}$, cells were scored for nuclear morphology of apoptosis (chromatin condensation, DNA fragmentation) by labeling with 4,6-diamidino-2-phenylindole (DAPI; Sigma 
Chemical Co., St. Louis, Missouri, USA), as described (16). Alternatively, infected cells were harvested at increasing time intervals (42-86 hours) and analyzed for DNA content by propidium iodide staining and flow cytometry, as described (16). In other experiments, transduced HeLa cells were cultivated for 20-60 hours at $37^{\circ} \mathrm{C}$ and assayed for caspase- 3 activity by hydrolysis of the fluorogenic substrate N-Acetyl-Asp-Glu-Val-Aspaldehyde (Ac-DEVD-AMC) (PharMingen, San Diego, California, USA), in the presence or absence of the caspase inhibitor Ac-DEVD-CHO. Caspase proteolytic processing was analyzed in the same cellular samples by Western blot analysis using a 1:5,000 dilution of a rabbit Ab to caspase-3 (Transduction Laboratories, Lexington, Kentucky, USA) or 1:1,000 dilution of an Ab to caspase-9 (Cell Signaling Technologies, Beverly, Massachusetts, USA), followed by chemiluminescence and autoradiography. For determination of mitochondrial release of cytochrome $c$, HeLa cells $\left(2 \times 10^{6}\right)$ infected with pAd-GFP or pAd-T34A (50 moi) for 8 hours at $37^{\circ} \mathrm{C}$ were harvested after an additional 6-12 hours of culture at $37^{\circ} \mathrm{C}$. Cells were washed in PBS, pH 7.4, and incubated for 30 minutes on ice in $300 \mu \mathrm{l}$ of lysis buffer containing $68 \mathrm{mM}$ sucrose, $200 \mathrm{mM}$ mannitol, $50 \mathrm{mM}$ $\mathrm{KCl}, 1 \mathrm{mM}$ EDTA, $1 \mathrm{mM}$ EGTA, $1 \mathrm{mM}$ DTT, and $1 \times$ complete protease inhibitor cocktail (Boehringer Mannheim Biochemicals Inc., Indianapolis, Indiana, USA). Cells were lysed with 80 strokes of a Dounce homogenizer using a type-B pestle and centrifuged at $800 \mathrm{~g}$ for 5 minutes at $4^{\circ} \mathrm{C}$. The supernatant was then collected and centrifuged at $14,000 \mathrm{~g}$ for 10 minutes at $4^{\circ} \mathrm{C}$. The supernatant (cytosol) and pellet (mitochondria) from this centrifugation were stored at $-70^{\circ} \mathrm{C}$ for Western blot analysis. Proteins from each sample were electrophoresed on a $15 \%$ SDS gel, transferred to nitrocellulose (BioRad Laboratories Inc., Hercules, California, USA), and analyzed by Western blotting with a mouse anti-cytochrome $c$ (7H8.2C12; PharMingen) $\mathrm{mAb}$ diluted to a concentration of 1:1,000. In another series of experiments, HeLa or MCF-7 cells were infected with pAd-GFP or pAd-T34A at moi of 50 for 8 hours at $37^{\circ} \mathrm{C}$ and further incubated in the presence or absence of chemotherapeutic drugs taxol $(2 \mu \mathrm{M}$; Sigma Chemical Co.) or adriamycin (100 nM; Sigma Chemical Co.). Aliquots of the various cultures were harvested at increasing time intervals between 24 and 96 hours and analyzed for DNA content by propidium iodide staining and flow cytometry, as described (16).

Adenoviral targeting of survivin in a xenograft breast cancer model. Six- to eight-week-old female CB17 SCID/beige mice (Taconic Farms, Germantown, New York, USA) were injected subcutaneously into the flanks with $1.5 \times 10^{6}$ exponentially growing MCF-7 cells in $250 \mu \mathrm{l}$ of sterile PBS, pH 7.4. Tumor growth was confined to local masses and did not affect animal survival over a 4-month observation period. In a first set of experiments, MCF-7 cells $\left(1.5 \times 10^{6}\right)$ were infected in vitro at a moi of 50 with pAd-T34A or pAd-GFP for 8 hours at $37^{\circ} \mathrm{C}$, washed, and injected into CB17
SCID mice. Tumor growth was measured in the three dimensions every other day and expressed as tumor volume in cubic millimeters. In a second set of experiments, animals were injected with uninfected MCF-7 cells $\left(1.5 \times 10^{6}\right)$ until local tumors became palpable after $7-10$ days ( $100-150 \mathrm{~mm}^{3}$ or $5-7 \mathrm{~mm}$ diameter). For local administration of pAd vectors, tumor masses were injected in three sites with pAd-GFP or pAdT34A at $5 \times 10^{8} \mathrm{GFU} /$ site of injection $(50 \mu \mathrm{l})$. Tumor growth was measured every other day for 2 weeks after viral injection. In a third series of experiments, 14 sixweek-old female SCID/beige mice received an intraperitoneal injection of uninfected exponentially growing $1.5 \times 10^{6} \mathrm{MCF}-7$ cells. After 7 days, mice were given a single intraperitoneal injection of $10^{10} \mathrm{GFU}$ of PAd-GFP or pAd-T34A (7 animals/group), in $150 \mu \mathrm{l}$ PBS, pH 7.4. After 4 days, animals were euthanized, and their total tumor burden was excised, weighed, and embedded in paraffin blocks for histology.

Histology. Tumors formed in animals injected with MCF-7 cells infected in vitro with pAd-GFP or pAdT34A were excised 30 days after transfer. Animals receiving intratumor injection of pAd-GFP or pAdT34A were sacrificed at 2,4 , and 7 days after pAd delivery, and tumors were removed for histology. For expression of pAd constructs in vivo, tumors were snap-frozen on dry ice in OCT, and cryostat sections were cut $(5 \mu \mathrm{m})$, placed on poly-L-lysine-coated slides, fixed with acetone for 10 minutes at $-20^{\circ} \mathrm{C}$, and analyzed for GFP expression by fluorescence microscopy. For histology, $5-\mu \mathrm{m}$ tissue sections from paraffin-embedded, formalin-fixed tumors were stained with hematoxylin-eosin (H\&E), or analyzed for reactivity with Ab's to the proliferation-specific marker Ki-67 (MIB-1; Zymed Laboratories Inc., South San Francisco, California, USA) or survivin by immunohistochemistry, as described (24). A proliferative index was obtained by counting the average number of Ki-67-positive cells in 8-9 high-power $(\times 400)$ fields, each containing approximately 600 cells. Tumor cell apoptosis was determined in vivo by internucleosomal DNA fragmentation by TUNEL staining, as described (18).

\section{Results}

Construction and expression of survivin adenoviruses. We used E. coli-based recombination of pAd-Track shuttle vectors (23) (Figure 1a) to obtain replication-deficient adenoviruses encoding WT survivin (pAd-WT) or a survivin $\mathrm{Thr}^{34} \rightarrow$ Ala mutant (pAd-T34A), abolishing the p34 ${ }^{\text {cdc2 }}$-cyclin B1 phosphorylation site (21). Infection of 293 cells with pAd-WT or PAd-T34A resulted in intense GFP labeling and strong induction of an approximately $16.5-\mathrm{kDa}$ survivin band as seen by Western blot analysis, as compared with pAd-GFP-infected cultures (not shown). Infection of cervical carcinoma HeLa or breast carcinoma MCF-7 cells with the various pAd vectors also resulted in intense GFP labeling in more than $85 \%$ of the cell population, as seen by fluo- 


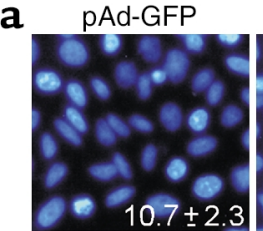

pAd-WT
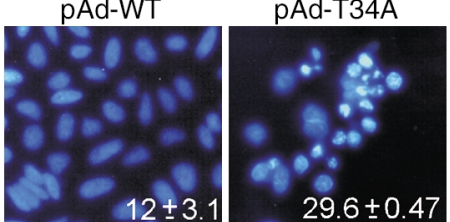

b
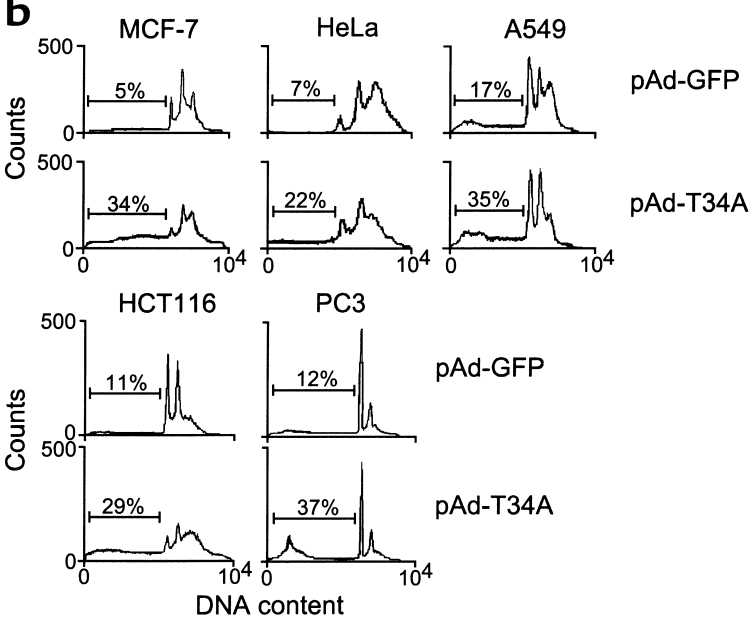

C

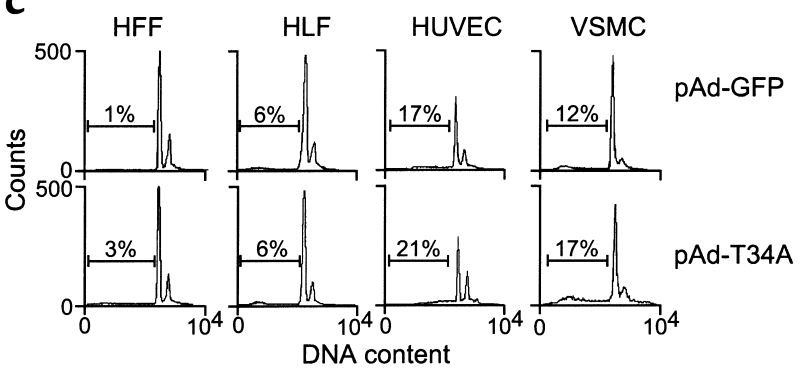

rescence microscopy (Figure 1b and data not shown). The potential contamination of viral stocks with replication-competent adenoviral particles was investigated. As shown in Figure 1c, successive infection of HeLa cells with cell extracts infected previously with pAd-WT or pAd-T34A at high moi $(1,250)$ resulted in negligible viral transduction, as determined by GFP expression (Figure 1c). As shown by Western blot analysis, infection of HeLa or MCF-7 cells with pAd-T34A was associated with appearance of a prominent approximately $16.5-\mathrm{kDa}$ survivin band, as compared with background levels of endogenous survivin in noninfected cells or cultures infected with pAd-GFP (Figure 1d). In addition, infection of HeLa or MCF-7 cells with pAd-GFP or pAd-T34A did not affect the endogenous levels of another antiapoptotic molecule, that is, XIAP, as seen by Western blot analysis (Figure 1d).

Selectivity of $p A d-T 34 A$ for induction of tumor cell apoptosis. Infection of HeLa cells with pAd-T34A caused morphologic features of apoptosis, including chromatin condensation and DNA fragmentation as seen by DAPI staining, as compared with pAd-GFP- or pAd-WT-treated cultures (Figure 2a). In cytofluorometric quantification of apoptosis, pAd-T34A induced a two- to threefold increase in the fraction of hypodiploid, i.e., apoptotic,

\section{Figure 2}

pAd-T34A selectively induces tumor cell apoptosis. (a) Nuclear morphology. HeLa cells infected with the indicated pAd vectors at moi of 50 for 8 hours were analyzed for nuclear apoptosis (chromatin condensation, DNA fragmentation) by DAPI staining. Numbers indicate the percentage of apoptotic cells under the various conditions tested. Data are the mean \pm SEM of three independent experiments. (b) Induction of tumor cell apoptosis by pAd-T34A. Aliquots of breast carcinoma MCF-7, cervical carcinoma HeLa, lung carcinoma A549, colorectal carcinoma HCT116, or prostate carcinoma PC3 were infected with pAd-GFP or pAd-T34A for 8 hours, harvested after 48-56 hours, and analyzed for DNA content by propidium iodide staining and flow cytometry. (c) Effect of pAd-T34A on normal cell viability. Human foreskin fibroblasts (HFF), human lung fibroblasts (HLF), human umbilical vein endothelial cells (HUVEC), or human vascular smooth muscle cells (VSMC) were infected with pAd-GFP or pAd-T34A and analyzed for DNA content by propidium iodide staining and flow cytometry, as described in $\mathbf{b}$. For $\mathbf{b}$ and $\mathbf{c}$, the percentages of apoptotic cells with hypodiploid (sub-G1) DNA content are indicated per each condition tested. Data are representative of one of two independent experiments with comparable results.

cells in cervical carcinoma HeLa, lung carcinoma A549, colorectal carcinoma HCT116, prostate carcinoma PC3, and a sevenfold increase in apoptosis in breast carcinoma MCF-7 cells, as compared with control cultures infected with pAd-GFP (Figure $2 \mathrm{~b}$ ). In contrast, pAdT34A did not significantly affect cell viability of proliferating normal human primary cell types, including HFFs and HLFs, HUVECs, or VSMCs, as seen by DNA content analysis and flow cytometry (Figure 2c).

Characterization of tumor cell apoptosis induced by pAdT34A. Infection of HeLa cells with pAd-T34A resulted in the appearance of an approximately $14-\mathrm{kDa}$ cytochrome $c$ band in cytosolic extracts, 6 hours after completion of viral infection, as seen by Western blot analysis (Figure 3a). This was associated with parallel reduction of cytochrome $c$ reactivity with mitochondrial fractions, 6 and 12 hours after viral infection (Figure 3a). In contrast, pAd-GFP did not cause increased detection of cytochrome $c$ in cytosolic extracts or reduction of cytochrome $c$ reactivity in mitochondrial fractions at the same time intervals after viral infection (Figure 3a). Treatment with pAd-T34A also resulted in timedependent proteolytic processing of approximately 46kDa proform caspase- 9 , which began to be detected 24 hours after viral infection and resulted in nearly complete disappearance of the proform caspase- 9 band by 96 hours, as seen by Western blot analysis (Figure 3b). This coincided with cleavage of approximately $32-\mathrm{kDa}$ caspase- 3 to active subunits of approximately 17 and 19 $\mathrm{kDa} 24$ hours after viral infection (Figure 3c) and increased caspase- 3 activity by DEVD hydrolysis, in a reaction entirely suppressed by the caspase-3 inhibitor, DEVD-CHO (Figure 3d). In control experiments, infection of HeLa cells with pAd-GFP did not cause caspase9 or caspase- 3 proteolytic processing as seen by Western blot analysis, and did not result in increased caspase-3 activity, as seen by DEVD hydrolysis (Figure 3, b-d). 


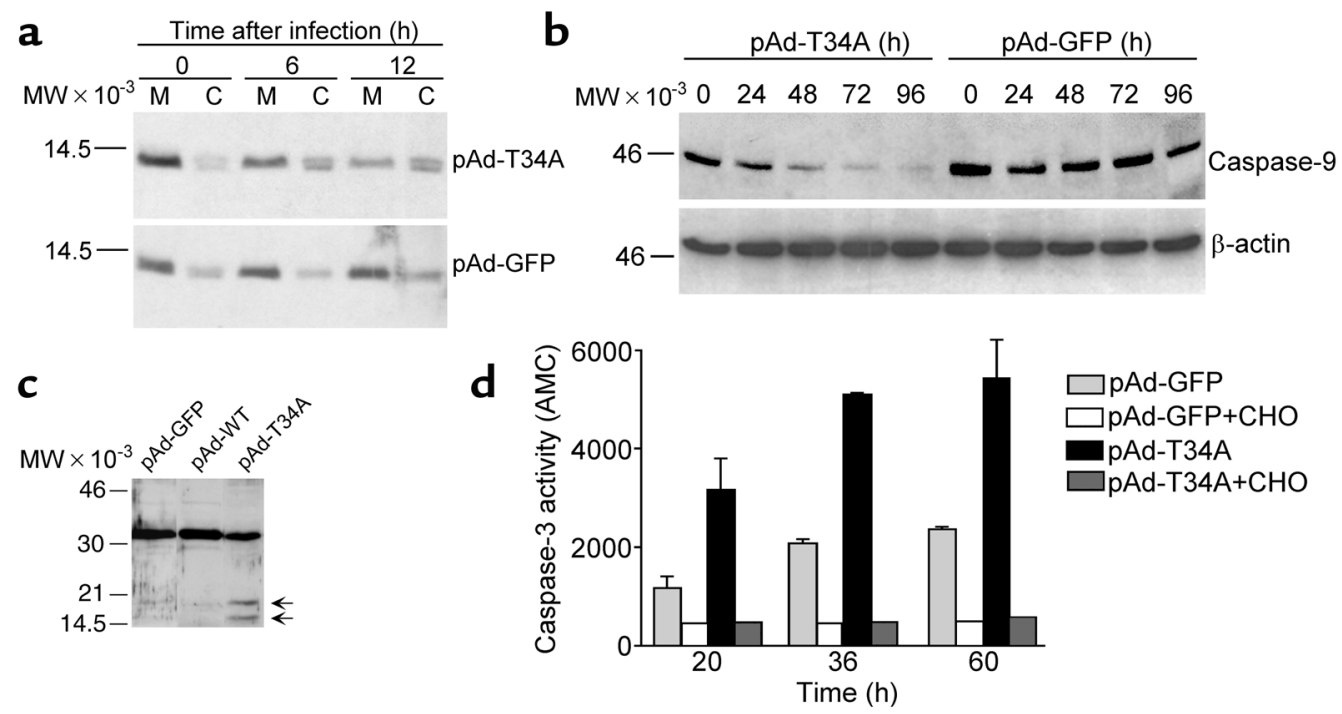

\section{Figure 3}

Characterization of pAd-T34A-induced tumor cell apoptosis. (a) Cytochrome $c$ release. HeLa cells were infected with pAd-GFP or pAd-T34A for 8 hours at $37^{\circ} \mathrm{C}$, harvested at the indicated time intervals followed by differential fractionation in cytosolic (C) and mitochondrial (M) extracts. Aliquots of each fraction were separated by SDS gel electrophoresis and analyzed for reactivity with an Ab to cytochrome $c$ by Western blotting. (b) Caspase-9 activation. The experimental conditions are the same as in a, except that detergent-solubilized extracts of HeLa cells infected with pAd-GFP or pAd-T34A were analyzed at the indicated time intervals for reactivity with an Ab to caspase- 9 by Western blotting. (c) Caspase-3 cleavage. HeLa cells were harvested 24 hours after infection with pAd-GFP or pAd-T34A and analyzed by Western blotting with an Ab to caspase- 3 . Arrows indicate the position of caspase- 3 active subunits of approximately 19 and $17 \mathrm{kDa}$. (d) Caspase-3 catalytic activity. Aliquots of HeLa cells infected with pAd-GFP or pAd-T34A were assayed at the indicated time intervals for caspase-3 activity by Ac-DEVD-AMC hydrolysis in the presence or absence of the caspase-3 inhibitor, DEVD-CHO. Data are the mean \pm SD of two independent experiments. Data are representative of one out of two to three independent experiments. For a-c, molecular-weight (MW) markers in kilodaltons are shown on the left.

Relationship between pAd-T34A-induced tumor cell apoptosis and cell cycle progression. Consistent with the data presented above, infection of HeLa cells with pAd-T34A resulted in a time-dependent increase in apoptotic cells with hypodiploid, that is, sub-G1 DNA content over an 86 hour time interval (Figure 4). This was also associated with progressive loss of the mitotic cell fraction with G2/M DNA content, in agreement with the kinetics of apoptosis at G2/M following inhibition of survivin phosphorylation at $\mathrm{Thr}^{34}(21)$. However, apoptosis induced by pAd-T34A was not preceded by cell cycle arrest or appearance of aneuploidy, as seen by DNA content analysis and flow cytometry (Figure 4). A small fraction of cells with more than 4N DNA content was indistinguishably observed in HeLa cells transduced with all pAd vectors and remained unchanged at all time points examined (Figure 4). In control experiments, HeLa cells infected with pAd-GFP or pAd-WT did not exhibit increased aneuploidy, and their background level of apoptosis remained unchanged throughout an 86-hour culture (Figure 4).

Effect of $p A d-T 34 A$ and chemotherapentic drugs on tumor cell apoptosis. Treatment of HeLa (Figure 5a) or MCF-7 cells (Figure $5 \mathrm{~b}$ ) with taxol resulted in cell cycle arrest at the metaphase-anaphase transition for 24-48 hours (not shown), followed by progressive appearance of apoptosis over a 72- to 96-hour time interval (Figure 5, $a$ and $b)$. In contrast, treatment with adriamycin result-

\section{Figure 4}

Relationship between pAd-T34A-induced tumor cell apoptosis and cell cycle progression. Aliquots of HeLa cells were infected with pAdGFP, pAd-WT, or pAd-T34A for 8 hours at $37^{\circ} \mathrm{C}$, harvested at the indicated time intervals, and analyzed for DNA content by propidium iodide staining and flow cytometry. The percentages of apoptotic cells with hypodiploid DNA content is indicated per each condition tested. Data are representative of one of two independent experiments with comparable results.

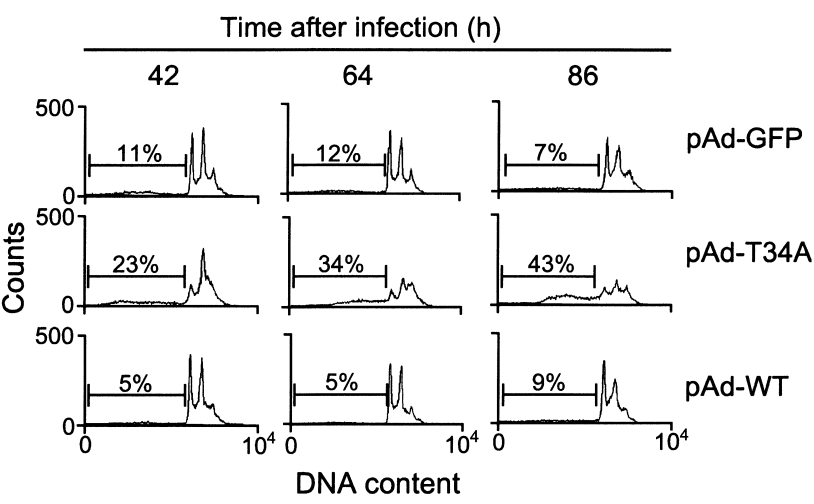



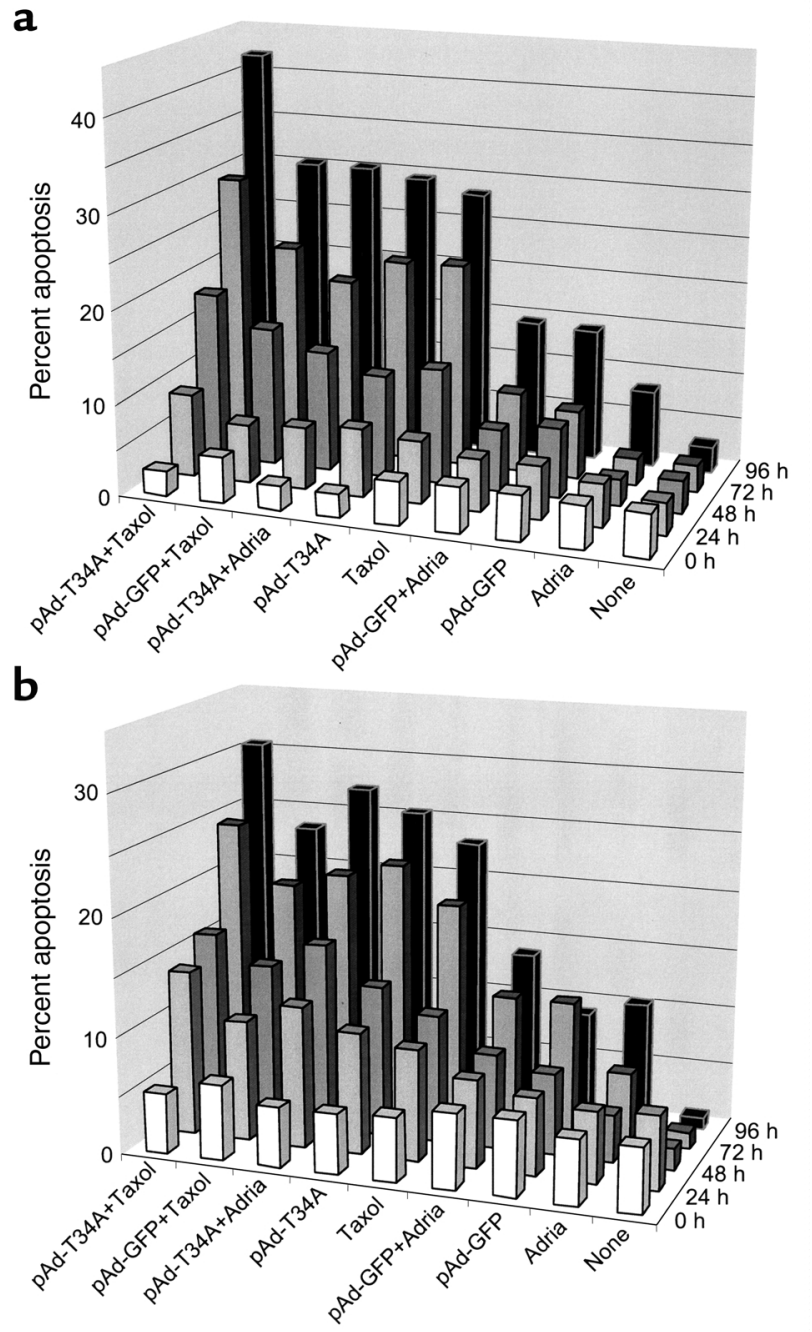

\section{Figure 5}

Effect of pAd-T34A and chemotherapeutic drugs on tumor cell apoptosis. Aliquots of HeLa (a) or MCF-7 (b) cells were treated with taxol $(2 \mu \mathrm{M})$ or adriamycin $(100 \mathrm{nM})$ in the presence or absence of pAd-GFP or pAd-T34A. Cells were harvested at the indicated time intervals and apoptosis was determined by DNA content analysis and flow cytometry as described in Figure 2. The time 0 hours corresponds to the first time point immediately after the 8-hour infection of HeLa or MCF-7 cells with the various pAd vectors. Data are representative of one of two independent experiments with comparable results.

ed in $\mathrm{G} 2 / \mathrm{M}$ arrest followed by only a modest increase in apoptosis detected after a 96-hour time interval in both HeLa or MCF-7 cells (Figure 5, a and b). Under these experimental conditions, administration of pAdT34A, but not pAd-GFP, was quantitatively as effective as taxol in time-dependent induction of apoptosis in both cell types and was considerably more effective than adriamycin treatment alone (Figure 5, a and b). When combined with chemotherapy, pAd-T34A enhanced taxol-induced apoptosis in both HeLa and MCF-7 cells at the longest time point examined, 96 hours, whereas the combination of pAd-T34A plus adriamycin was not more effective than pAd-T34A treatment alone (Figure 5, a and b). In control experi- ments, addition of pAd-GFP did not increase apoptosis induced by taxol or adriamycin in HeLa or MCF-7 cells (Figure 5, a and b).

Modulation of tumor formation and tumor growth by $p$ AdT34A. Injection of uninfected MCF-7 cells in SCID mice resulted in the formation of localized tumors 7-12 days after transfer into the animals (Figure 6a). Ex vivo infection of MCF-7 cells with pAd-T34A, but not pAd-GFP, before transfer into the animals suppressed the formation of macroscopic tumors in SCID mice (Figure 6a, see below). Histologically, MCF-7 tumors were composed of nodules of malignant epithelial cells, which stained intensely positive for endogenous survivin as seen by immunohistochemistry (Figure 6b). When analyzed for kinetics of tumor growth, uninfected MCF-7 cells or MCF-7 cells infected with pAd-GFP gave rise to comparable exponentially growing tumors over a 30-day period (Figure 6c). In contrast, all animals reconstituted with MCF-7 cells infected ex vivo with pAd-T34A remained tumor free for the first 21 days, after which slow-growing, small tumors began to appear in 8 out of the 15 animals tested (Figure 6c). MCF-7 tumors formed under these conditions were harvested after 30 days and analyzed for histology. Control tumors originated from pAd-GFP-infected MCF-7 cells exhibited intense labeling for the proliferation-associated marker $\mathrm{Ki}-67$, as seen by immunohistochemistry (Figure 6d). In contrast, only scattered and faintly positive Ki-67-positive cells were detected in tumors originated from pAdT34A-infected MCF-7 cells (Figure 6d). Quantification of Ki-67-positive cells under these conditions revealed that initial infection with pAd-T34A resulted in a more than $90 \%$ inhibition of mitotic index, as compared with pAd-GFP tumors (Figure 6e).

Next, we tested the effect of pAd-T34A on established subcutaneous tumors. Uninfected MCF-7 cells were injected into the flank of SCID mice, and tumors were allowed to reach a volume of approximately $100-150 \mathrm{~mm}^{3}$ over a 7 - to 10 -day period $(5-7 \mathrm{~mm}$ diameter). Injection of these tumors in three sites with pAd-GFP or pAd-T34A at $5 \times 10^{8} \mathrm{GFU} /$ site resulted in the appearance of numerous GFP-expressing cells along the needle injection tracks (Figure 7a), as seen by fluorescence microscopy of frozen tumor sections. Intratumor administration of pAd-GFP did not affect exponential tumor growth for up to 14 days after injection (Figure $7 \mathrm{~b}$ ). In contrast, a single intratumor administration of pAd-T34A inhibited tumor growth by approximately $40 \%$ at day $7,9,11$, and 14 after injection (Figure $7 \mathrm{~b}$ ). Tumors were harvested 2 days after local injection of pAd vectors and analyzed for histology. By H\&E staining, putative intratumor needle injection tracks were visible after local administration of pAd-GFP or pAd-T34A (Figure 7c). Tumors injected with pAd-GFP contained mitotic cells as determined by Ki-67 labeling (Figure 7b) and exhibited no reactivity for in situ apoptosis by TUNEL staining along the needle injection tracks (Figure 7c). In con- 


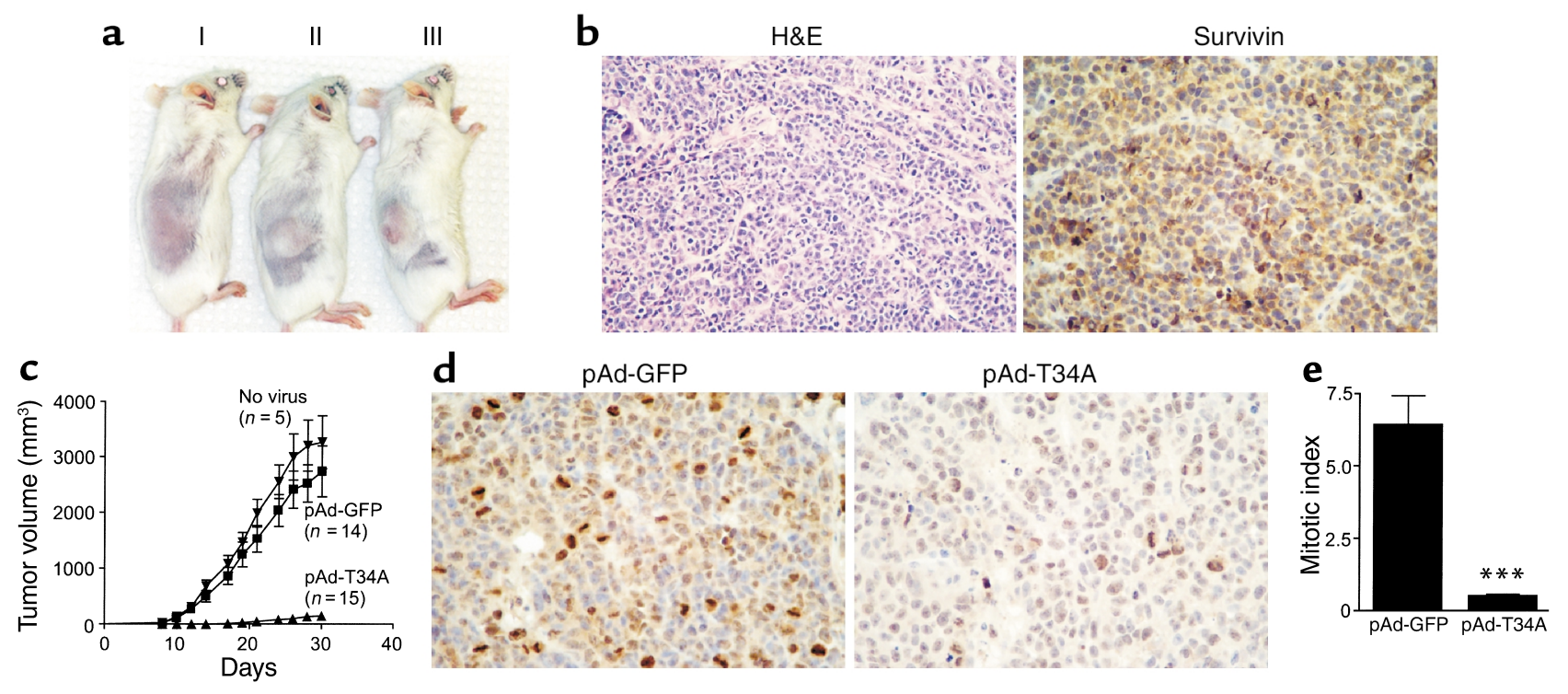

Figure 6

Inhibition of tumor formation by pAd-T34A. (a) Xenograft breast cancer model. MCF-7 cells $\left(1.5 \times 10^{6}\right)$ were infected at moi of 50 with pAd-T34A (I), control pAd-GFP (II), or left untreated (III) for 8 hours, and injected subcutaneously in CB17 female SCID/beige mice. Macroscopic tumors were detected within 7-12 days from transfer into the animals. (b) Histology. Tissue sections of formalin-fixed, paraffinembedded MCF-7 tumors were stained by H\&E or analyzed for survivin expression by immunohistochemistry. (c) Kinetics of tumor formation. The experimental conditions are as in $\mathbf{a}$. Tumor growth was monitored in three dimensions and expressed as tumor volume in cubic millimeters. Data are the mean \pm SEM of the various animals per group indicated in parenthesis. (d) Inhibition of tumor cell proliferation by pAd-T34A. Tumor sections from animals receiving MCF-7 cells infected ex vivo with pAd-GFP or pAd-T34A were analyzed for reactivity with the proliferation-specific Ab Ki-67 by immunohistochemistry. (e) Mitotic index in MCF-7 tumors. The experimental conditions are as in $\mathbf{d}$. A proliferative index was obtained by counting the average number of Ki-67-positive cells in eight to nine high-power $(\times 400)$ fields, each containing approximately 600 cells. ${ }^{*} * P<0.0001$.

trast, tumors injected with pAd-T34A exhibited loss of Ki-67 reactivity and strong labeling for internucleosomal DNA fragmentation by TUNEL along the needle injection tracks (Figure 7c).

The effect of PAd-T34A on a model of disseminated peritoneal tumor growth was also investigated. For these experiments, exponentially growing uninfected MCF-7 cells were injected intraperitoneally in SCID mice. After a week, animals were given a single intraperitoneal injection of pAd-GFP or pAd-T34A, sacrificed 4 days later, and subjected to necroscopic examination. Four out of seven animals given pAd-GFP contained peritoneal masses ranging from 8.6 to $95.8 \mathrm{mg}$ in weight (Table 1 ), which were individually confirmed as human breast tumors by H\&E staining (not shown). In contrast, only two out of seven animals treated with pAd-T34A contained small intraperitoneal tumors of 6.5 and $14 \mathrm{mg}$ in weight (Table 1). This resulted in a mean total tumor burden of $140.1 \mathrm{mg}$ for pAdGFP-treated animals versus $20.5 \mathrm{mg}$ for SCID mice receiving pAd-T34A (Table 1 ).

\section{Discussion}

In this study we have shown that a replication-deficient adenovirus encoding a phosphorylation-defective survivin $\mathrm{Thr}^{34} \rightarrow$ Ala mutant ( $\left.\mathrm{pAd}-\mathrm{T} 34 \mathrm{~A}\right)$ caused spontaneous apoptosis in various tumor cell lines, exhibited no toxicity for normal human cells, and inhibited tumor growth in three distinct xenograft breast cancer models, in vivo.

Adenovirus vectors targeting regulators of cell cycle and/or apoptosis have been investigated for their potential suitability in cancer gene therapy alone or to enhance the efficacy of chemotherapy (25-29). In this context, encouraging results were obtained using an E1B gene-deleted replication-selective oncolytic adenovirus, that is, ONYX-015 (30), in the treatment of patients with head and neck cancer, especially when combined with chemotherapy $(31,32)$. A similar approach also involved intratumor adenoviral delivery

\section{Table 1}

Effect of pAd-T34A on disseminated peritoneal tumors

\begin{tabular}{lcc}
\hline Animals & pAd-GFP & pAd-T34A \\
1 & $20.4 \mathrm{mg}$ & None \\
2 & $95.8 \mathrm{mg}$ & None \\
3 & None & $6.5 \mathrm{mg}$ \\
4 & $15.3 \mathrm{mg}$ & None \\
5 & $8.6 \mathrm{mg}$ & $14.0 \mathrm{mg}$ \\
6 & None & None \\
7 & None & None \\
Total tumor weight & $140.1 \mathrm{mg}$ & $20.5 \mathrm{mg}$
\end{tabular}

SCID/beige animals were injected intraperitoneally with $1.5 \times 10^{6} \mathrm{MCF}-7$ cells followed 7 days later by a single intraperitoneal injection of pAd-GFP or pAdT34A ( $\left.10^{10} \mathrm{GFU}\right)$. Animals (7/group) were sacrificed after 4 days, subjected to necroscopy, and peritoneal tumors under the various treatments were removed, weighted, and confirmed histologically by H\&E staining. 
a

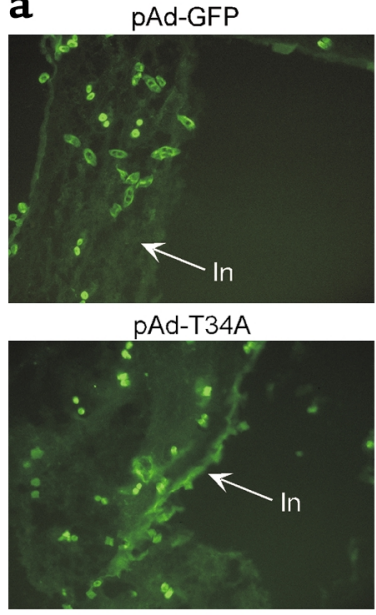

b

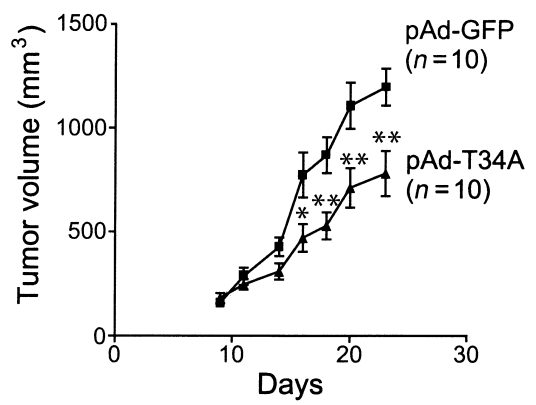

C

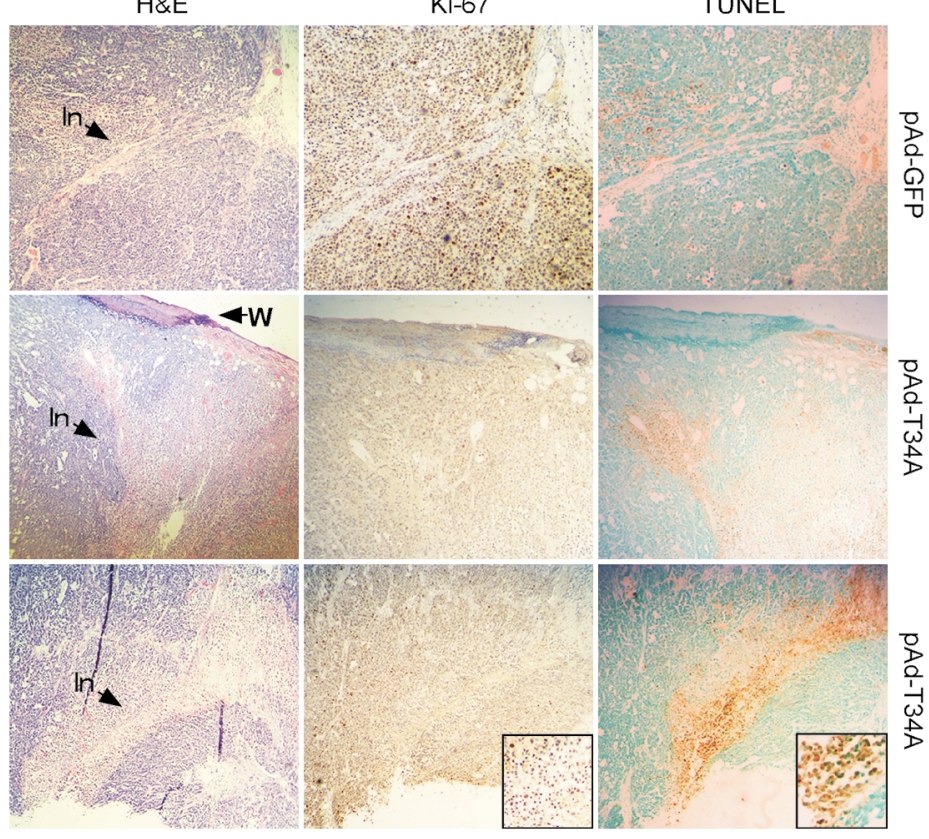

Figure 7

Effect of intratumor injection of PAd-T34A on tumor growth, proliferation, and apoptosis. (a) Expression of injected pAd vectors in vivo. Uninfected MCF-7 cells $\left(1.5 \times 10^{6}\right)$ were injected into the flank of female CB17 $\mathrm{SCID} /$ beige mice, and tumors were allowed to grow to approximately $100-150 \mathrm{~mm}^{3}$ volume (7-10 mm diameter). Tumors were injected with pAd-GFP or pAd-T34A in three sites $\left(5 \times 10^{8} \mathrm{GFU} /\right.$ site $)$ and harvested after 48 hours ( $p A d-T 34 A$ ) or 96 hours ( $\mathrm{pAd}-\mathrm{GFP}$ ). Cryostat tumor sections were analyzed for GFP expression by fluorescence microscopy. In, needle injection track. $\times 200$. (b) Kinetics of tumor growth. The experimental conditions are as in a. Tumor growth was measured in three dimensions at the indicated time intervals after intratumor injection of pAd-GFP or pAd-T34A. Data are the mean \pm SEM of the various animals per group indicated in parenthesis. ${ }^{*} P<0.05,{ }^{*} P<0.005$. (c) Histology. Tumor sections from one animal injected with PAd-GFP or two representative animals injected with PAd-T34A were analyzed after 48 hours by $\mathrm{H} \& \mathrm{E}$ staining $(\mathrm{H} \& \mathrm{E})$, cell proliferation by Ki-67 labeling (Ki-67), or in situ apoptosis by TUNEL labeling (TUNEL). In, putative needle injection tracks; W, epidermal wound corresponding to the putative entry site of injection. $\times 100$. Insets show loss of Ki-67-positive cells and strong TUNEL labeling along the needle injection tracks of pAdT34A-treated tumors. Insets, $\times 200$. of WT p53 in patients with non-small cell lung cancer to restore checkpoint functions of apoptosis and/or cell cycle arrest $(33,34)$. As presented here, there are several aspects of adenoviral targeting of the survivin pathway that may be desirable for cancer gene therapy. First, expression of pAd-T34A was sufficient to directly induce apoptosis in five different cancer cell types. This did not require the integrity of the $\mathrm{p} 53 / \mathrm{Rb}$ checkpoint (preserved in MCF-7 and HCT116 cells, but functionally or genetically inactivated in HeLa, PC3, and A549 cells) and was not affected by the constitutive expression of other apoptosis inhibitors in tumor cells, that is, XIAP. Secondly, when judged against mainstay cancer therapy, the magnitude of tumor cell apoptosis induced by PAd-T34A was comparable to taxol and significantly more robust than adriamycin. Current protocols for cancer gene therapy typically involve combination with chemotherapy $(31,32)$, and data presented here suggest that pAd-T34A plus taxol, but not plus adriamycin, enhanced tumor cell apoptosis. For the expression of survivin at mitosis and its inhibition of taxol-induced apoptosis (8), it is possible that interference with survivin function may be therapeutically more efficacious when selectively combined with drugs targeting the $\mathrm{G} / 2 \mathrm{M}$ transition. Lastly, and perhaps most importantly, expression of PAd-T34A did not affect cell viability or cell cycle progression of normal human cells, including fibroblasts, VSMCs, and endothelium. Because a mitotic fraction was clearly evident in the DNA content analysis of these cells, the data presented here make it unlikely that expression of pAd-T34A results in a generic toxicity for all proliferating cells, whether normal or transformed. Taken together with the low to undetectable level of survivin in normal tissues as opposed to cancer in vivo $(7,10)$ and the lack of macroscopic toxicity following systemic administration of pAd-T34A in vivo (see below), these data suggest that adenoviral targeting of the survivin pathway may provide a considerable degree of selectivity for tumor cells and potential limited toxicity for normal tissues in vivo.

The mode by which survivin counteracts apoptosis and, conversely, the mechanism of cell death ensuing from manipulation of the survivin pathway have been the subject of intense investigation $(35,36)$. The ability of pAd-T34A to cause mitochondrial release of cytochrome $c$ in tumor cells, followed by proteolytic processing of caspase-9, cleavage of caspase-3, and increased caspase- 3 catalytic activity, suggests that survivin may control a step in 
the initiation/amplification of the mitochondrial (intrinsic) apoptotic cascade. This is consistent with the formation of a survivin-caspase-9 complex in mitotic cells (21) and the fact that apoptosis induced by plasmid expression of survivin $\mathrm{Thr}^{34} \rightarrow$ Ala was entirely reversed by a caspase- 9 , but not a caspase- 8 dominant negative mutant (21). On the other hand, we cannot presently exclude that cytochrome $c$ release induced by pAd-T34A may result from increased autocatalytic activity of caspase-9 with cleavage of cytosolic factor(s) acting on mitochondrial permeability (37).

$\mathrm{Thr}^{34}$ in survivin constitutes a phosphorylation site for the main mitotic kinase p34 $4^{\text {cdc2 }}$-cyclin B1 (21), and it has been speculated that this region may be ideally suited to modulate the binding of potential antiapoptotic ligands to the survivin baculovirus IAP repeat in a phosphorylation-dependent manner (36). Previous studies demonstrated that survivin $\mathrm{Thr}^{34} \rightarrow$ Ala mutant retained the ability to physically associate with $\mathrm{p} 34^{\mathrm{cdc} 2}$ cyclin B1 (21) and prevent phosphorylation of endogenous survivin (18). This suggests that pAd-T34A may operate with a similar dominant negative mechanism and promote tumor cell apoptosis by interfering with the kinetics of survivin phosphorylation at mitosis. On the other hand, this does not result in a global interference of $\mathrm{p} 34^{\mathrm{cdc} c}$ kinase activity, as shown by the ability of pAd-T34A-infected cells to progress through the cell cycle and complete mitosis similarly to pAdGFP-treated cultures. In addition, at variance with adenoviral delivery of Cdk inhibitors (25) and contrary to the suggestions of knockout studies (38), apoptosis induced by pAd-T34A did not result from defects in cell cycle progression or appearance of aneuploidy.

With respect to the antitumor effect of pAd-T34A in vivo, this cannot be ascribed to an inflammatory response to viral infection because fully immunodeficient animals were used in these models. The lack of antitumor activity by pAd-GFP also rules out any "adenoviral" effects attributable to Ad sequences, as suggested for pAd-p53 (39), and, similarly, plasmid expression of survivin $\mathrm{Thr}^{34} \rightarrow$ Ala lacking Ad sequences (18, $21)$ induced tumor cell apoptosis in vitro and in vivo similarly to pAd-T34A. In a first xenograft model of tumor formation, all animals receiving MCF-7 cells infected ex vivo with pAd-T34A remained tumor free for 21 days, after which small, slow-growing tumors began to appear in 8 of the 15 animals tested. Consistent with the anticipated half-life of adenoviral vectors in vivo (40), this may reflect clearance of PAd-T34A, or, alternatively, re-entry into the cell cycle of a previously quiescent fraction of MCF-7 cells. On the other hand, the loss of Ki-67-positive cells in these tumors suggests that any residual pAd-T34A effectively inhibited tumor cell proliferation even 3 weeks after viral transfer. In a second xenograft model of tumor treatment, a single intratumor injection of PAd-T34A inhibited the growth of established tumors by approximately $40 \%$, which is comparable to the local administration of pAd p53 or ONYX-015 in similar models $(39,41)$. Although analy- sis of GFP-labeled cells confirmed that pAd-T34A or pAd-GFP exclusively infected along the needle injection tracks, as expected for a replication-deficient adenovirus (42), this was associated with massive apoptosis in situ and loss of proliferating cells, thus potentially explaining the impairment of tumor growth. A similar model may also apply to the ability of PAd-T34A to inhibit disseminated intraperitoneal tumor growth, potentially adding flexibility for future protocols of adenoviral delivery for tumor treatment in vivo.

In summary, these data suggest that pAd-T34A may provide a novel approach for cancer gene therapy, selectively promoting tumor cell apoptosis, sparing normal tissues, and potentially enhancing the efficacy of chemotherapeutic drugs operative at mitosis. These studies may be relevant for novel therapeutic strategies aimed at dysregulating the apoptotic balance in cancer and improve the efficacy of chemotherapy-induced apoptosis in patients (20).

\section{Acknowledgments}

We thank B. Vogelstein for providing pAd vectors and J. S. Schechner for reviewing histology data. This work was supported by NIH grants CA-78810, CA-90917 (D.C. Altieri), and HL-10112 (M. Mesri).

1. Hanahan, D., and Weinberg, R.A. 2000. The hallmarks of cancer. Cell.
100:57-70.

2. Hengartner, M.O. 2000. The biochemistry of apoptosis. Nature. 407:770-776.

3. Reed, J.C. 1999. Dysregulation of apoptosis in cancer. J. Clin. Oncol. 17:2941-2953.

4. Soengas, M.S., et al. 2001. Inactivation of the apoptosis effector Apaf-1 in malignant melanoma. Nature. 409:207-211.

5. Kroemer, G., and Reed, J.C. 2000. Mitochondrial control of cell death. Nat. Med. 6:513-519.

6. Deveraux, Q.L., and Reed, J.C. 1999. IAP family proteins-suppressors of apoptosis. Genes Dev. 13:239-252.

7. Ambrosini, G., Adida, C., and Altieri, D.C. 1997. A novel anti-apoptosis gene, survivin, expressed in cancer and lymphoma. Nat. Med. 3:917-921.

8. Li, F., et al. 1998. Control of apoptosis and mitotic spindle checkpoint by survivin. Nature. 396:580-584.

9. Reed, J.C., and Bischoff, J.R. 2000. BIRinging chromosomes through cell division: and survivin' the experience. Cell. 102:545-548.

10. Velculescu, V.E., et al. 1999. Analysis of human transcriptomes. Nat. Genet. 23:387-388.

11. Monzo, M., et al. 1999. A novel anti-apoptosis gene: re-expression of survivin messenger RNA as a prognosis marker in non-small-cell lung cancers. J. Clin. Oncol. 17:2100-2104.

12. Swana, H.S., Grossman, D., Anthony, J.N., Weiss, R.M., and Altieri, D.C. 1999. Tumor content of the antiapoptosis molecule survivin and recurrence of bladder cancer. N. Engl. J. Med. 341:452-453.

13. Tanaka, K., et al. 2000. Expression of survivin and its relationship to loss of apoptosis in breast carcinomas. Clin. Cancer Res. 6:127-134.

14. Adida, C., et al. 2000. Prognostic significance of survivin expression in diffuse large B-cell lymphomas. Blood. 96:1921-1925.

15. Kato, J., et al. 2001. Expression of survivin in esophageal cancer: correlation with the prognosis and response to chemotherapy. Int. J. Cancer. 95:92-95.

16. Li, F., et al. 1999. Pleiotropic cell-division defects and apoptosis induced by interference with survivin function. Nat. Cell Biol. 1:461-466.

17. Chen, J., et al. 2000. Down-regulation of survivin by antisense oligonucleotides increases apoptosis, inhibits cytokinesis and anchorage-independent growth. Neoplasia. 2:235-241.

18. Grossman, D., Kim, P.J., Schechner, J.S., and Altieri, D.C. 2001. Inhibition of melanoma tumor growth in vivo by survivin targeting. Proc. Natl. Acad. Sci. USA. 98:635-640.

19. Olie, R.A., et al. 2000. A novel antisense oligonucleotide targeting survivin expression induces apoptosis and sensitizes lung cancer cells to chemotherapy. Cancer Res. 60:2805-2809.

20. Nicholson, D.W. 2000. From bench to clinic with apoptosis-based therapeutic agents. Nature. 407:810-816 
21. O'Connor, D.S., et al. 2000. Regulation of apoptosis at cell division by p34 cdc2 phosphorylation of survivin. Proc. Natl. Acad. Sci. USA. 97:13103-13107.

22. O'Connor, D.S., et al. 2000. Control of apoptosis during angiogenesis by survivin expression in endothelial cells. Am. J. Pathol. 156:393-398.

23. He, T.C., et al. 1998. A simplified system for generating recombinant adenoviruses. Proc. Natl. Acad. Sci. USA. 95:2509-2514.

24. Grossman, D., McNiff, J.M., Li, F., and Altieri, D.C. 1999. Expression and targeting of the apoptosis inhibitor, survivin, in human melanoma. J. Invest. Dermatol. 113:1076-1081.

25. Schreiber, M., Muller, W.J., Singh, G., and Graham, F.L. 1999. Comparison of the effectiveness of adenovirus vectors expressing cyclin kinase inhibitors p16INK4A, p18INK4C, p19INK4D, p21(WAF1/CIP1) and p27KIP1 in inducing cell cycle arrest, apoptosis and inhibition of tumorigenicity. Oncogene. 18:1663-1676.

26. Gurnani, M., Lipari, P., Dell, J., Shi, B., and Nielsen, L.L. 1999. Adenovirus-mediated p53 gene therapy has greater efficacy when combined with chemotherapy against human head and neck, ovarian, prostate, and breast cancer. Cancer Chemother. Pharmacol. 44:143-151.

27. Arafat, W.O., et al. 2000. An adenovirus encoding proapoptotic Bax induces apoptosis and enhances the radiation effect in human ovarian cancer. Mol. Ther. 1:545-554.

28. Griffith, T.S., Anderson, R.D., Davidson, B.L., Williams, R.D., and Ratliff, T.L. 2000. Adenoviral-mediated transfer of the TNF-related apoptosisinducing ligand/Apo-2 ligand gene induces tumor cell apoptosis. $J$. Immunol. 165:2886-2894.

29. Steinwaerder, D.S., et al. 2001. Tumor specific gene expression in hepatic metastases by a replication-activated adenovirus vector. Nat. Med. 7:240-243.

30. Bischoff, J.R., et al. 1996. An adenovirus mutant that replicates selectively in p53-deficient human tumor cells. Science. 274:373-376.

31. Khuri, F., et al. 2000. A controlled trial of intratumoral ONYX-015, a selectively-replicating adenovirus, in combination with cisplatin and 5 fluorouracil in patients with recurrent head and neck cancer. Nat. Med. 6:879-995.

32. Nemunaitis, J., et al. 2001. Phase II trial of intratumoral administration of ONYX-015, a replication-selective adenovirus, in patients with refractory head and neck cancer. J. Clin. Oncol. 19:289-298.

33. Swisher, S.G., et al. 1999. Adenovirus-mediated p53 gene transfer in advanced non-small-cell lung cancer. J. Natl. Cancer Inst. 91:763-771.

34. Weill, D., et al. 2000. Adenoviral-mediated p53 gene transfer to nonsmall cell lung cancer through endobronchial injection. Chest. 118:966-970.

35. Shin, S., et al. 2001. An anti-apoptotic protein human survivin is a direct inhibitor of caspase-3 and -7. Biochemistry. 40:1117-1123.

36. Verdecia, M.A., et al. 2000. Structure of the human anti-apoptotic protein survivin reveals a dimeric arrangement. Nat. Struct. Biol. 7:602-608.

37. Bossy-Wetzel, E., and Green, D.R. 1999. Caspases induce cytochrome c release from mitochondria by activating cytosolic factors. J. Biol. Chem. 274:17484-17490.

38. Uren, A.G., et al. 2000. Survivin and the inner centromere protein INCENP show similar cell- cycle localization and gene knockout phenotype. Curr. Biol. 10:1319-1328.

39. Nielsen, L.L., et al. 1997. Efficacy of p53 adenovirus-mediated gene therapy against human breast cancer xenografts. Cancer Gene Ther. 4:129-138.

40. Hamada, K., et al. 1996. Adenovirus-mediated transfer of a wild-type p53 gene and induction of apoptosis in cervical cancer. Cancer Res. 56:3047-3054

41. Heise, C.C., Williams, A.M., Xue, S., Propst, M., and Kirn, D.H. 1999. Intravenous administration of ONYX-015, a selectively replicating adenovirus, induces antitumoral efficacy. Cancer Res. 59:2623-2628.

42. Kurihara, T., Brough, D.E., Kovesdi, I., and Kufe, D.W. 2000. Selectivity of replication-competent adenovirus for human breast carcinoma cells expressing the MUC1 antigen. J. Clin. Invest. 106:763-771. 